\title{
Improved Efficiency of Ternary-Blended Films in Organic Solar Cell
}

\author{
Received 16 December, 2020; revised 25 December, 2020; accepted 30 December, 2020
}

\author{
Eunhee $\operatorname{Lim}^{a, *}$ \\ Department of Chemistry, Kyonggi University, Suwon 16227, Republic of Korea
}

*Corresponding author E-mail: ehlim@kyonggi.ac.kr

\begin{abstract}
Organic solar cells (OSCs) have attracted great interest as next-generation energy sources. In this study, a diketopyrrolopyrrole (DPP)-based electron-rich small-molecule donor, $\alpha$-DPP-DT, was incorporated into a P3HT:PCBM binary film to fabricate ternary-blended OSCs. The OSCs were fabricated with the conventional configuration of ITO/PEDOT:PSS/active layer/LiF/Al and exhibited a power conversion efficiency of $2.12 \%$, which was greater than those of the two corresponding binary-blended devices. Both the open-circuit voltage and short-circuit current density values of the ternary films were lay between the corresponding values of the two binary films. The proposed working mechanism of the ternary-blended films is based on an alloy structure of two donors rather than on a cascade structure.
\end{abstract}

Keywords: Organic solar cell, Organic photovoltaic cell, Ternary cell

\section{Introduction}

Advances in various materials and in device manufacturing technology have led to the development of high-performance organic solar cells (OSCs) $[1,2]$. With respect to materials development, new molecular backbones and synthesis strategies, such as halogen substitution and side-chain engineering, have been widely introduced. For example, power conversion efficiencies (PCEs) greater than $12 \%$ have been achieved using nonfullerene small-molecule acceptors composed of a fused-ring-based electron-rich core and suitable halogen substituents [3]. The development of new molecular backbones can lead to next-level device performance, which can be further increased through fine-tuning of the side-chains/substituents. Since the development of Y6 and COi8DFC, device performance has improved by more than $16 \%[4,5]$. With respect to advances in manufacturing, the introduction of ternary and/or tandem devices has contributed to improvements in device performance [6,7]. Unlike binary devices, which comprise one donor and one acceptor, ternary solar cells include a third component (donor or acceptor) in their active layer. This configuration is generally used to improve photovoltaic performance. The third component used in a binary system must satisfy several requirements to enhance the performance of solar cells, including appropriate molecular energy levels, a good film morphology, and complementary absorption. The introduced third component can affect charge transfer of the films because of the energy offset of the other materials used in the device, which influences the open-circuit voltage $\left(V_{\mathrm{OC}}\right)$. Control of the film roughness and morphology is necessary because improper morphological characteristics can induce phase separation of the photoactive layer. Complementary UV-vis absorption of the three materials enables the absorption of a broader range of the solar spectrum, which can improve the short-circuit current density $\left(J_{\mathrm{SC}}\right)$ of solar cell devices. For commercialization, further improvement of device performance is still required; to this end, the development of new materials and the optimization of devices in which they are incorporated must be considered simultaneously.

In this study, a ternary system was developed by incorporat- ing a diketopyrrolopyrrole (DPP)-based small-molecule donor as the third component into a poly(3-hexylthiophene) (P3HT):phenyl- $\mathrm{C}_{61}$ butyric acid methyl ester (PCBM) binary system. Device performance was improved upon incorporation of the third component. A working mechanism was also proposed.

\section{Experimental details}

\subsection{Materials}

$\alpha$-DPP-DT was synthesized using the palladium-catalyzed Suzuki coupling reaction between the DPP-based dibromide, 2, 5diethylhexyl-3,6-bis (5-bromothiophen-2-yl) pyrrolo[3,4-c]-pyrrolo1,4-dione, and the borolanylaryl compound, 5-hexylthiophene2-boronic acid pinacol ester [8]. DPP-based dibromide was synthesized according to the literatures [9-11], and the borolanylaryl compound was commercially available from Sigma-Aldrich.

\subsection{Physical measurements and fabrication of or-} ganic solar cells

UV-vis spectra were obtained using a Shimadzu UV/vis spectrometer. The films used in the UV-vis measurement were prepared by drop-casting a chlorobenzene solution. The optical energy bandgaps $\left(E_{\mathrm{g}}\right)$ were estimated from the absorption onset wavelengths $\left(E_{\mathrm{g}}=\right.$ $\left.1240 / \lambda_{\text {onset }}(\mathrm{eV})\right)$ of the films [8]. The electrochemical properties were characterized by cyclic voltammetry $(\mathrm{CV})$, as presented in our previous reports $[8,9]$.

The OSC devices were fabricated with the conventional configuration: indium tin oxide (ITO)/poly(3, 4-ethylenedioxythiophene) polystyrene sulfonate (PEDOT:PSS)/active layer/LiF/Al. The ITOcoated glass substrates were cleaned via ultrasonic treatment in deionized water, acetone, isopropyl alcohol, and methanol. The PEDOT:PSS layer $(40 \mathrm{~nm})$ was spin-coated on the substrate at a rate of $3500 \mathrm{rpm}$ for $30 \mathrm{~s}$ and annealed at $120{ }^{\circ} \mathrm{C}$ for $30 \mathrm{~min}$. In the ternary cells, both P3HT and $\alpha$-DPP-DT were used as a donor, and the PCBM 

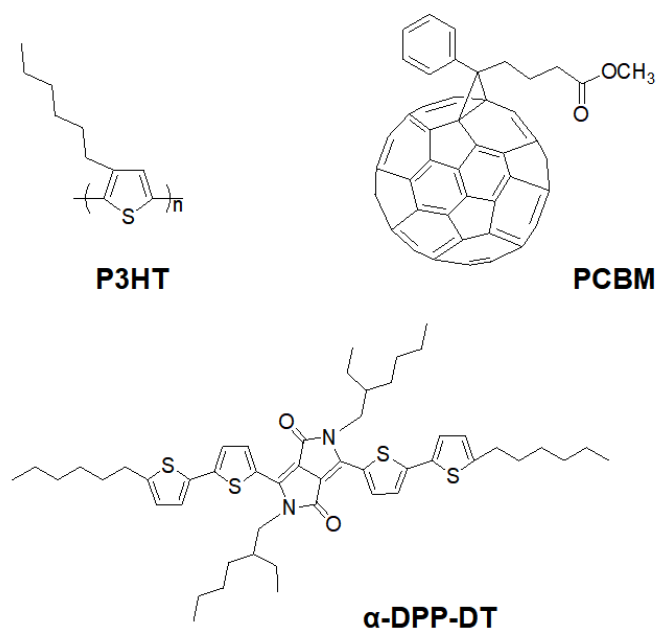

Figure 1. Molecular chemical structures of alpha-DPP-DT, P3HT, and PCBM

was used as an acceptor. The ternary-blended solution (P3HT: $\alpha$-DPPDT:PCBM $=8: 2: 8$ ) was prepared using chlorobenzene as a solvent at a solid concentration of $36 \mathrm{mg} \mathrm{mL}^{-1}$. For comparison, a P3HT:PCBM binary cell was also prepared under the same conditions, where the D:A ratio was adjusted to 10:8, and chlorobenzene was used as a processing solvent. Prior to use, the chlorobenzene solution was stirred at $60^{\circ} \mathrm{C}$ overnight to ensure complete dissolution. The solutions were filtered through a $0.45 \mu \mathrm{m}$ polytetrafluoroethylene membrane syringe before applied dropwise onto the substrates. The active layer was then spin-coated onto the PEDOT:PSS-coated ITO substrates at a rate of $3000 \mathrm{rpm}$ for $30 \mathrm{~s}$, followed by soft-baking at $120^{\circ} \mathrm{C}$ for $10 \mathrm{~min}$ in a nitrogen-filled glove box. The thickness of the photoactive layer was approximately $50 \mathrm{~nm}$, measured using a surface profiler (Alpha-Step 200, Tencor). Subsequently, an $\mathrm{LiF}(1 \mathrm{~nm}) / \mathrm{Al}(100 \mathrm{~nm})$ electrode was then thermally evaporated on the active layer under vacuum. The effective area of all the devices was measured to be $4 \mathrm{~mm}^{2}$. The currentvoltage $(J-V)$ characteristics of the devices were measured using a Keithley 2400 source-measure unit in air under AM 1.5G illumination $\left(100 \mathrm{~mW} \mathrm{~cm}^{-2}\right)$.

\section{Results and discussion}

\subsection{Materials and physical properties}

Well-known $p$ - and $n$-type organic semiconducting materials P3HT and PCBM were used as a donor and an acceptor, respectively, constituting the active layer of an OSC. The electron-rich DPP-based small molecule $\alpha$-DPP-DT was selected as another donor material to be incorporated into the active layer, resulting in a ternary-blended film of P3HT: $\alpha$-DPP-DT:PCBM. We reported the synthesis of $\alpha$-DPPDT via a Pd-catalyzed Suzuki coupling reaction between DPP-based dibromide and a hexylthiophene-based borolanylaryl compound in our previous work $[8,9]$. Figure 1 shows the chemical structure of $\alpha$-DPP-DP along with the structures of P3HT and PCBM.

Figure 2 shows the UV-vis absorption spectrum of the ternary P3HT: $\alpha$-DPP-DT:PCBM film, along with the spectra of the P3HT:PCBM and $\alpha$-DPP-DT:PCBM binary films. The P3HT: $\alpha-$ DPP-DT:PCBM weight ratio was adjusted to $8: 2: 8$, which is the same ratio used in the fabricated device, as described later. In addition, when P3HT and $\alpha$-DPP-DT were used as donors and PCBM as an acceptor, the total donor:acceptor weight ratio in the ternary devices was 10:8; thus, the binary P3HT:PCBM film was also fabricated with a weight ratio of 10:8. For convenience, the three absorption spectra were normalized to maximum values of

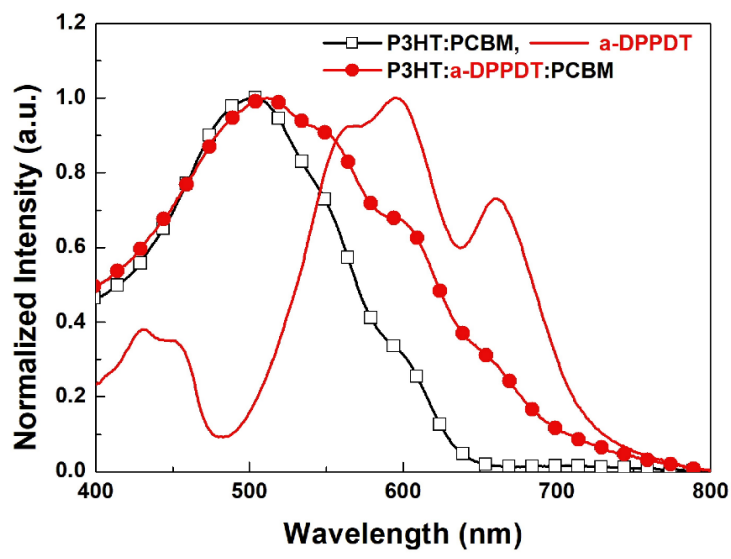

Figure 2. Normalized UV-vis absorption spectra of the P3HT: $\alpha$-DPPDT:PCBM (8:2:8) ternary-blended films; those of P3HT:PCBM (10:8) and $\alpha$-DPPDT films are shown for comparison.

1. In the spectrum of the binary film composed of P3HT and PCBM, the maximum absorption peak appears at $503 \mathrm{~nm}$. P3HT is a well-known wide-bandgap polymer donor. The spectrum of the ternary-blended film of P3HT: $\alpha$-DPP-DT:PCBM exhibits an absorption maximum peak at $512 \mathrm{~nm}$, which is slightly red-shifted but similar to that of the spectrum of the P3HT:PCBM film. In addition, the spectrum of the P3HT: $\alpha$-DPP-DT:PCBM film exhibits a shoulder peak at $\sim 600 \mathrm{~nm}$, which corresponds to the maximum absorption of the $\alpha$-DPP-DT film. Given the blend ratio of the ternary film (P3HT: $\alpha$-DPP-DT:PCBM $=8: 2: 8$ ), the addition of a relatively small amount of $\alpha$-DPP-DT ( $10 \%$ of the total) into P3HT:PCBM substantially red-shifted and broadened the UV-vis absorption peak of the resultant ternary film. The absorption edge in the long-wavelength region shifted from $640 \mathrm{~nm}$ (P3HT:PCBM) to $720 \mathrm{~nm}$ (P3HT: $\alpha$-DPP-DT:PCBM), accompanied by the appearance of a long absorption tail that extended to $800 \mathrm{~nm}$. Such broad and red-shifted UV-vis absorption behavior is known to be advantageous in absorbing light in the photoactive layer of OSCs.

Figure 3 shows the energy diagram of the three materials used in the ternary solar cells; this diagram depicts the highest occupied molecular orbital (HOMO), as measured by $\mathrm{CV}$, the calculated optical $E_{\mathrm{g}}$, and the lowest unoccupied molecular orbital (LUMO) energy levels. $E_{\mathrm{g}}$ of $\alpha$-DPP-DT was $1.72 \mathrm{eV}$, which is relatively lower than that of the wide-bandgap polymer donor P3HT $(2.0 \mathrm{eV})$. Importantly, the incorporation of $\alpha$-DPP-DT into the P3HT:PCBM resulted in cascade energy-level alignment. The LUMO level of $\alpha$-DPP-DT $(-3.59 \mathrm{eV})$ was between the LUMO levels of P3HT $(-3.2 \mathrm{eV})$ and PCBM $(-4.2$ $\mathrm{eV})$. Similarly, the HOMO level $(-5.2 \mathrm{eV})$ of $\alpha-\mathrm{DPP}-\mathrm{DT}$ was between the HOMO levels of P3HT and PCBM; thus cascade charge transfer could occur in the ternary solar cells.

\subsection{Organic solar cells}

The OSC devices were fabricated using the conventional configuration ITO/PEDOT:PSS $(40 \mathrm{~nm}) /$ active layer $(50 \mathrm{~nm}) / \mathrm{LiF}(1 \mathrm{~nm}) / \mathrm{Al}$

Table 1. Photovoltaic properties of the organic solar cells.

\begin{tabular}{lcccc}
\hline Active layer & $V_{\mathrm{OC}}[\mathrm{V}]$ & $\begin{array}{c}J_{\mathrm{SC}}[\mathrm{mA} \\
\left.\mathrm{cm}^{-2}\right]\end{array}$ & $F F[\%]$ & PCE [\%] \\
$\begin{array}{l}\text { P3HT: } \alpha \text { - } \\
\text { DPPDT:PCBM }\end{array}$ & 0.64 & 6.44 & 51 & 2.12 \\
$\begin{array}{l}\alpha \text {-DPPDT:PCBM } \\
\text { [8] }\end{array}$ & 0.79 & 5.29 & 42 & 1.77 \\
P3HT:PCBM & 0.57 & 7.28 & 44 & 1.83 \\
\hline
\end{tabular}




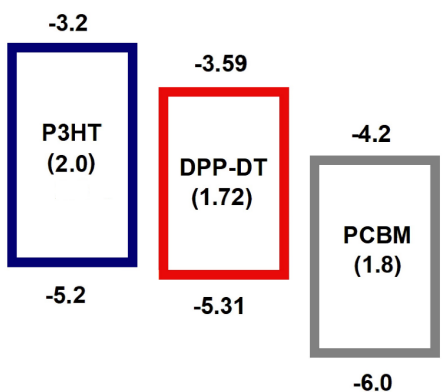

Figure 3. Energy diagrams of $\alpha$-DPP-DT, P3HT, and PCBM

$(100 \mathrm{~nm})$. Figure 4 shows the $J-V$ curves recorded in air under AM $1.5 \mathrm{G}$ illumination $\left(100 \mathrm{~mW} \mathrm{~cm} \mathrm{~cm}^{-2}\right)$, and the photovoltaic properties of the devices are summarized in Table 1; the photovoltaic properties of the two binary devices with P3HT:PCBM (10:8) and $\alpha$-DPP-DT:PCBM (2:1) [8] films are included for comparison. The ternary device fabricated using the P3HT: $\alpha$-DPP-DT:PCBM (8:2:8) blend film exhibited substantially enhanced performance (PCE of 2.12 $\%)$ compared with the two binary P3HT:PCBM (1.83 \%) and $\alpha$-DPPDT:PCBM (1.77\%) devices.

Interestingly, both the $V_{\mathrm{OC}}$ and $J_{\mathrm{SC}}$ values of the device with the ternary film were between the corresponding values of the devices fabricated using the two binary films. First, the $J_{\mathrm{SC}}$ value of the P3HT: $\alpha$ DPP-DT:PCBM blend film device $\left(6.44 \mathrm{~mA} \mathrm{~cm}^{-2}\right)$ was higher than that of the $\alpha$-DPP-DT:PCBM film device $\left(5.29 \mathrm{~mA} \mathrm{~cm}^{-2}\right)$ but lower than that of the P3HT:PCBM film device $\left(7.28 \mathrm{~mA} \mathrm{~cm}^{-2}\right)$. Despite the relatively low $E_{\mathrm{g}}$ of $\alpha$-DPP-DT and the cascade energy-level alignment of the three materials [Fig. 3], the addition of $\alpha$-DPPDT resulted in a lower charge transport in the device. Therefore, the working mechanism of the ternary-blended film might not involve a cascade structure. The $V_{\mathrm{OC}}$ value of the ternary film device was also between those of the device with the two binary films. The $V_{\mathrm{OC}}$ values increased in the order P3HT:PCBM, ternary film, and $\alpha$-DPP-DT:PCBM. The higher $V_{\mathrm{OC}}$ value of the $\alpha$-DPP-DT:PCBM film device $(0.79 \mathrm{~V})$ compared with that of the P3HT:PCBM film device $(0.57 \mathrm{~V})$ is consistent with the LUMO levels of the donor materials. The $V_{\mathrm{OC}}$ value of the OSC device is known to be dominated by the difference in the HOMO level of the donor and LUMO level of the acceptor. Therefore, given the LUMO level of the acceptor, PCBM, the lower-lying HOMO level of $\alpha$-DPP-DT $(-5.31 \mathrm{eV})$ compared with that of P3HT $(-5.2 \mathrm{eV})$ could make $V_{\mathrm{OC}}$ of the $\alpha$-DPP-DT:PCBM device $(0.79 \mathrm{~V})$ higher than that of the P3HT:PCBM device $(0.57 \mathrm{~V})$. The gradually varying $V_{\mathrm{OC}}$ dependence on the content of the third component has been explained previously using an alloy-like model $[12,13]$. In our device, the intermediate $V_{\mathrm{OC}}$ value of the ternary device indicates that the two donors adopted an alloy structure rather than a cascade structure. In addition, the improved fill factor $(F F)$ value of $51 \%$ for the ternary-blended device implies the formation of an improved film morphology, where the addition of $\alpha$-DPP-DT does not cause substantial phase separation. The device efficiency can presumably be further improved by optimizing the device fabrication conditions.

\section{Conclusions}

This study improved the device performance of OSCs by incorporating a small-molecule donor, alpha-DPP-DT, as the third component into the OSC active layer. The ternary-blended P3HT: $\alpha$-DPPDT:PCBM device exhibited a PCE of $2.12 \%$, which is superior to those of devices based on the corresponding blended films of P3HT:PCBM $(1.83 \%)$ and alpha-DPP-DT:PCBM $(1.77 \%)$. Because the $V_{\text {OC }}$ value

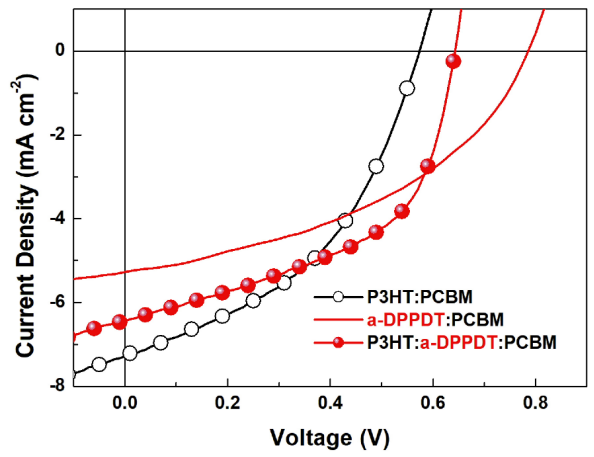

Figure 4. J-V curves of the DPP-DT-based on binary and ternary films

was between those of the corresponding two binary devices, the working mechanism of the ternary cells was attributed to the formation of an alloy-like structure.

\section{Acknowledgements} 2018.

This work was supported by Kyonggi University Research Grant

\section{References}

[1] H. Wang, J. Cao, J. Yu, Z. Zhang, R. Geng, L, Yang, and W. Tang, J. Mater. Chem. A 7, 4313 (2019).

[2] C. Zhao, Y. Guo, Y. Zhang, N. Yan, S. You, and W. Li, J. Mater. Chem. A 7, 10174 (2019).

[3] J. Wang, S. Xie, D. Zhang, R. Wang, Z. Zheng, H. Zhou, and Y. Zhang, J. Mater. Chem. A 6, 19934 (2018).

[4] J. Lee, A. R. Han, J. Kim, Y. Kim, J. H. Oh, and C. Yang, J. Am. Chem. Soc. 134, 20713 (2012).

[5] L. Wu, L. Xie, H. Tian, R. Peng, J. Huang, B. Fanady, W. Song, S. Tan, W. Bi, and Z. Ge, Sci. Bull. 64, 1087 (2019).

[6] D. Di Carlo Rasi, and R. A. J. Janssen, Adv. Mater. 31, 1806499 (2019).

[7] P. Bi and X. Hao, Solar RRL 3, 1800263 (2019).

[8] E. Lim, Bull. Kor. Chem. Soc. 41, 639 (2020).

[9] E. Lim, S. Lee, K. K. Lee, I. N. Kang, S. J. Moon, H. Y. Kong, and H. E. Katz, Sol. Energy Mater. Sol. Cells 107, 165 (2012).

[10] Y. Kim, C. E. Song, A. Cho, J. Kim, Y. Eom, J. Ahn, S. J. Moon, and E. Lim, Mater. Chem. Phys. 143, 825 (2014).

[11] E. Ripaud, D. Demeter, T. Rousseau, E. Boucard-Cétol, M. Allain, R. Po, P. Leriche, and J. Roncali, Dyes Pigment. 95, 126 (2012).

[12] Q. An, J. Wang, W. Gao, X. Ma, Z. Hu, J. Gao, C. Xu, M. Hao, X. Zhang, C. Yang, and F. Zhang, Sci. Bull. 65, 538 (2020).

[13] Q. An, J. Wang, and F. Zhang, Nano Energy 60, 768 (2019). 